\title{
Édentement unitaire : réhabilitation par bridge collé
}

\section{RÉSUMÉ}

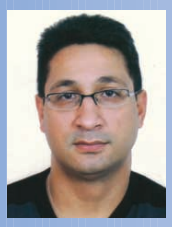

Hicham SOUALHI

Chirurgien-dentiste spécialiste en prothèse fixée.

La chaumiere $2, n^{\circ} 30$,

Hay ryad, Rabat - Maroc.

\section{Amal EL YAMANI}

Professeur d'enseignement supérieur en prothèse fixée.

Jamal EL BERNOUSSI

Professeur d'enseignement supérieur en prothèse fixée,

Chef de service de prothèse fixée,

Faculté de médecine dentaire,

Rabat - Maroc.

L'édentement unitaire représente l'une des situations cliniques qui offre de nombreuses solutions thérapeutiques. Quel que soit le secteur concerné, les hypothèses de traitement sont nombreuses : prothèse implanto-portée, bridge collé, bridge conventionnel, prothèse amovible ou même traitement orthodontique.

Dans les limites de leur champ d'indication, les bridges collés constituent une alternative de choix répondant aux impératifs fonctionnels, esthétiques et biologiques.

Ils s'adressent pour l'essentiel à des cas d'édentement unitaire, b rdé par des dents en normoposition, presque saines et de mobilité réduite. La rigueur de leur indication constitue un élément important dans leur succès.

- édentement unitaire

- prothèse collée

- décision thérapeutique 


\section{Introduction}

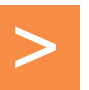

Devant un cas d'édentement unitaire, si les solutions implanto-portées représentent souvent une thérapeutique convoitée, dans certaines situations, des contraintes d'ordre médical, anatomique ou financier ne nous permettent pas toujours d'envisager ce choix thérapeutique. Un bridge collé représente alors une alternative de choix répondant aux impératifs fonctionnels, esthétiques et biologiques.

Durant cette dernière décennie, l'évolution de nouveaux matériaux a permis l'émergence et la réactualisation des nouvelles propositions de traitement ; parmi ces propositions réactualisées, la prothèse collée utilisant les progrès réalisés dans le domaine de l'adhésion ainsi que le développement de la notion d'économie tissulaire.

Un bridge collé est une pièce prothétique rigide liée par collage aux piliers afin de remplacer une ou voire deux dents absentes. II est défini comme étant une structure métallique composée d'une pontique reliée à deux ailettes qui seront collées sur les faces palatines des dents adjacentes à l'édentement (fig. 1).

Dès 1972, Rochette a initié la réalisation de la prothèse collée. L'intérêt majeur de ce type de reconstitution était d'éviter une mutilation excessive des dents supports et d'introduire la notion de réversibilité en cas d'échec. L'absence de préparation dentaire, très séduisante dans les premières années, a été nuancée face aux échecs répétés. Ainsi le collage ne dispense pas de préparations, lui seul ne peut pas remplacer la qualité de rétention, ni la résistance aux forces occlusales de descellement et de cisaillement assurés par les préparations.

Aujourd'hui, il est unanimement admis qu'une préparation a minima des piliers est indispensable pour assurer la pérennité de la prothèse collée [1].

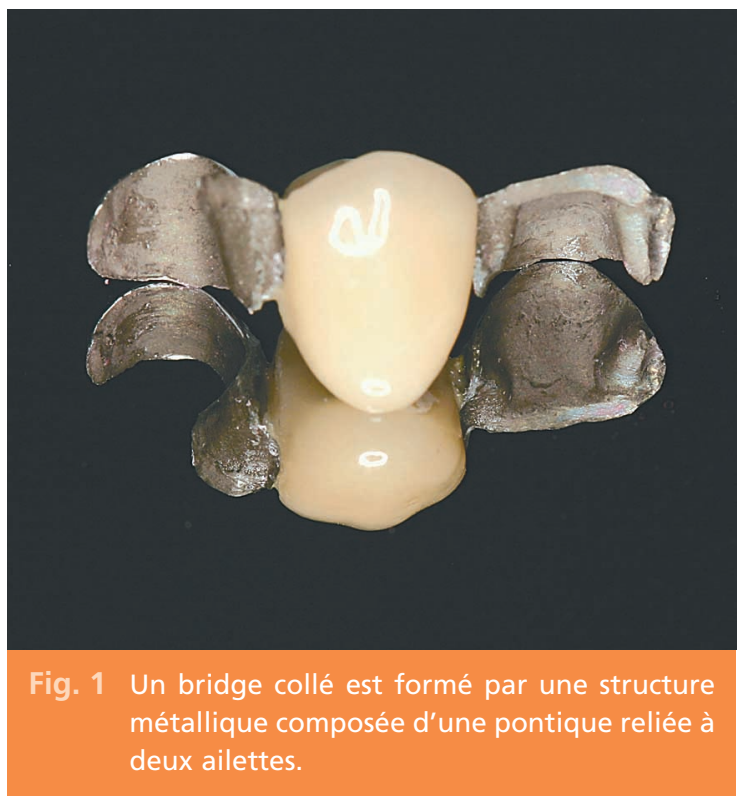




\section{Édentement unitaire et bridge collé}

La meilleure option thérapeutique est subordonnée à une analyse précise du contexte clinique. Cette analyse permet d'aboutir à un choix final raisonné, elle fait intervenir des éléments anatomophysiologiques, des éléments psychologiques et sociaux (motivation, coopération, moyens) et enfin des éléments de compétence de la part du praticien.

\section{Facteurs en rapport \\ avec le patient}

La prothèse adhésive doit être réservée à des patients conscients que leur hygiène est un facteur conditionnant le succès à moyen terme de leur prothèse. En plus, d'autres éléments sont à considérer :

- l'état de santé général : la situation médicale du patient peut constituer une contradiction majeure à certaines solutions thérapeutiques; ainsi, pour les patients ne pouvant subir de chirurgie, le traitement implantaire sera à proscrire ;

- l'âge : pour la prothèse collée, le taux d'échec est souvent plus élevé chez les patients jeunes. Cela est attribué à des facteurs morphologiques, anatomiques et chimiques [2]. Cependant, I'incidence biologique, les risques pulpaires et le taux d'échecs également élevé d'une construction conventionnelle chez ces jeunes patients impliquent de préférer une construction collée même si son pronostic est limité dans le temps.

Pour le traitement implantaire, la croissance constitue un facteur défavorable pour une restauration implanto-portée, l'ensemble implant ostéointégré ne peut suivre la croissance du procès alvéolaire au fur et à mesure des modifications des structures craniofaçiales. Ainsi la prothèse partielle collée (sans préparation des dents piliers) peut représenter un traitement provisoire ;

- la motivation et les moyens financiers du patient : le coût financier ainsi que la disponibilité du patient ont une influence directe dans le choix thérapeutique. Ainsi, si la disponibilité n'est pas suffisante, la mise en œuvre d'un traitement nécessitant un délai d'exécution long est contre-indiquée.

\section{Facteurs prothétiques}

\section{> Facteurs dentaires}

Plusieurs éléments sont à évaluer. L'évaluation des dents bordant l'édentement guide largement le choix thérapeutique. Le choix judicieux consiste à compenser l'édentement en préservant l'intégrité des dents adjacentes [3]. Ainsi, si les dents adjacentes sont partiellement atteintes par des lésions carieuses ou porteuses de restaurations extensives, leur utilisation en tant que pilier de bridge conventionnel est tout à fait justifiée. Si elles sont au contraire parfaitement saines, la préservation des tissus est primordiale.

C'est alors l'indication d'une solution thérapeutique la moins mutilante possible : implantologie, prothèse amovible. La prothèse partielle collée constitue une solution intermédiaire en termes de mutilation tissulaire.

La forme de la dent doit être évaluée. La hauteur de la couronne clinique (indice de Le Huche: différence en $\mathrm{mm}$ entre le plus grand diamètre mésio-distal de la dent et le diamètre mésio-distal au niveau cervical de la préparation) doit être faible : il faut éviter les 

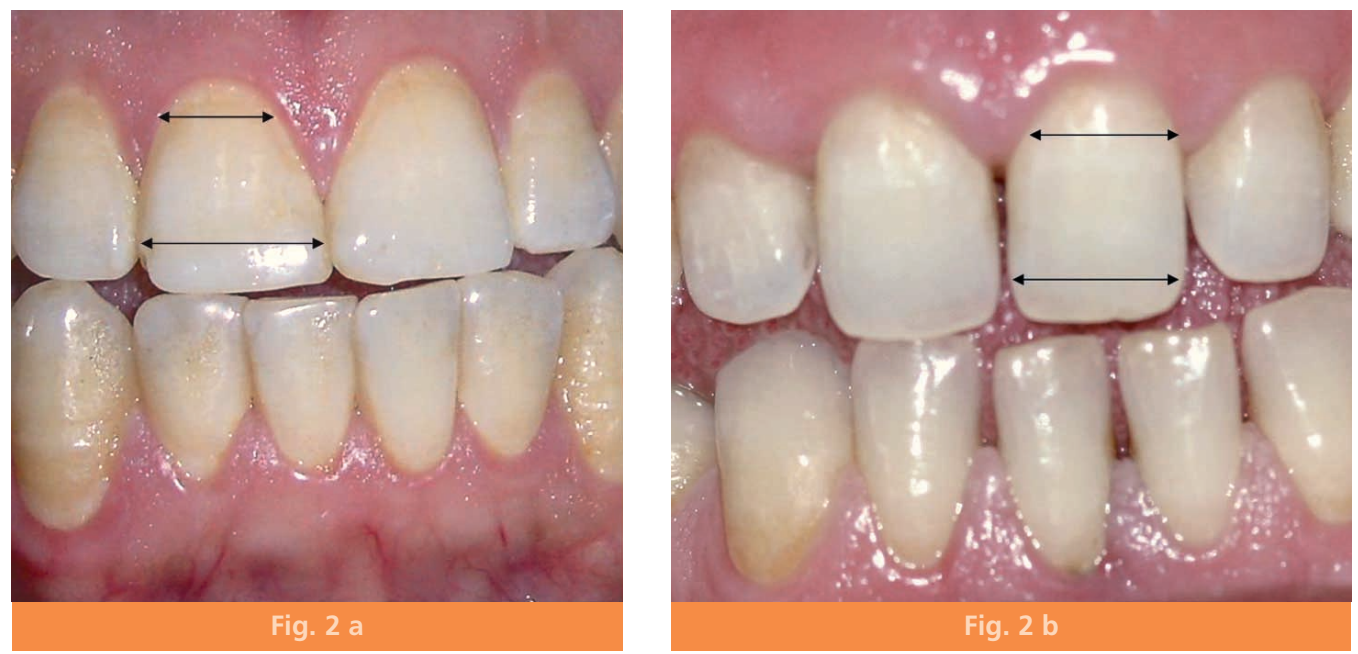

Fig. 2 a et b L'indice de Le Huche correspond à la différence entre la largueur maximum de la couronne et la largueur du collet dans le sens mésio-distal (d'après Shillingburg 1998).

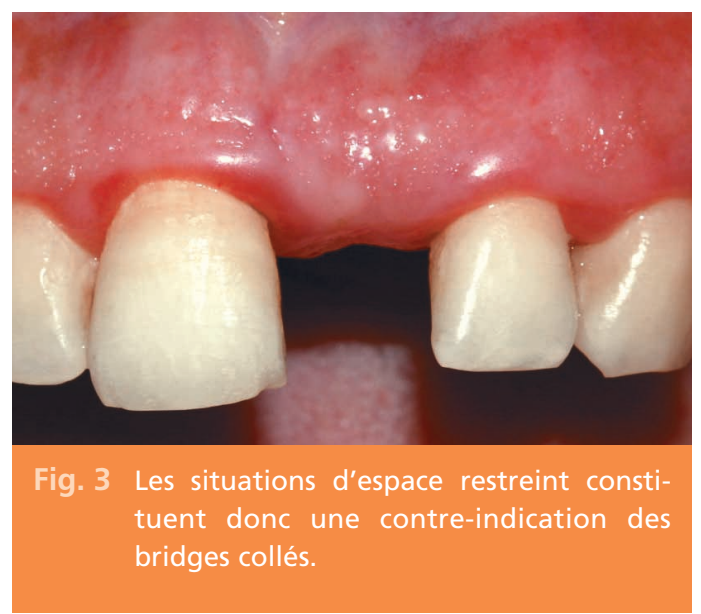

dents triangulaires (fig. 2 a et b). Les dents courtes limitent la surface de collage. Des piliers ménageant des hauteurs suffisantes de préparation seront indiqués [4].

Les dents piliers doivent être en position orthocentrée. Des dents supports en malposition nécessitent une accentuation des préparations afin de les paralléliser. Cela aurait pour conséquence d'obtenir une surface de collage en grande partie dentinaire, mais également une proximité pulpaire pouvant amener à dépulper ultérieurement.

\section{> Longueur d'édentement}

Le bridge collé est indiqué dans les édentements de très faible étendue : une dent, exceptionnellement deux (incisives inférieures) [5].

La place disponible nécessite un examen attentif, car il n'est pas possible de modifier de façon simple et fiable la largeur apparente de l'intermédiaire du bridge ou des dents piliers comme cela est possible avec la prothèse conventionnelle [6]. Les situations d'espace restreint ou important constituent donc un facteur limitant l'indication de la prothèse partielle collée (fig. 3). 


\section{$>$ Parodonte}

Une mobilité dentaire excessive liée à une faiblesse parodontale est une contre-indication aux bridges collés [7]. Elle modifie les conditions d'application des contraintes de l'assemblage entre la dent et l'élément prothétique et entraîne une sollicitation importante du joint collé pouvant entraîner son décollement.

\section{$>$ Facteurs occlusaux}

Les surcharges occlusales et les parafonctions sont des contre-indications des prothèses collées [8]. Les contraintes qu'elles occasionnent conditionnent la stabilité de l'élément prothétique. Des rapports interarcades non traumatogènes constituent les meilleures situations des constructions collées.

\section{Indications}

Le bridge collé s'adresse pour l'essentiel à des cas d'édentement unitaire, bordé par des dents en normoposition, presque saines et de mobilité réduite (tableau I).
Généralement, ces indications constituent le plus souvent des situations cliniques idéales pour la réalisation d'une prothèse unitaire implanto-portée. Cependant, le verdict en

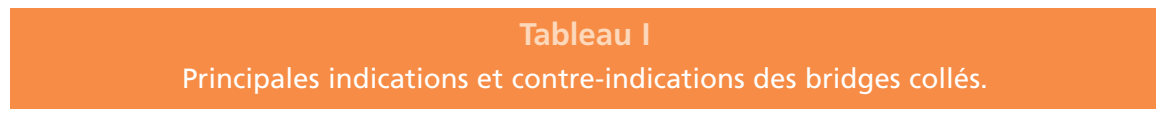

\begin{tabular}{|c|c|c|}
\hline & Situations favorables & Situations défavorables \\
\hline État général & $\begin{array}{c}\text { Cardiopathie } \\
\text { avec un risque infectieux majeur }\end{array}$ & \\
\hline Hygiène & Irréprochable & Défectueuse \\
\hline Largeur de l'édentement & $\begin{array}{l}\text { - Remplacement d'1 dent absente } \\
\text { - Remplacement de } 2 \text { incisives } \\
\text { mandibulaires }\end{array}$ & $\begin{array}{l}\text { - Espace d' } 1 \text { dent absente } \\
\text { en présence de diastème } \\
\text { - Remplacement de plus de } 2 \text { dents }\end{array}$ \\
\hline \multicolumn{3}{|l|}{ Examen des dents piliers } \\
\hline Volume coronaire & Important & Faible \\
\hline - Carie & Absente ou peu étendue & Importante \\
\hline - Qualité de l'émail & Bonne & Dysplasie (problème de collage) \\
\hline - Pulpe & Rétraction pulpaire & Volumineuse \\
\hline - Indice MD de Le Huche & Faible & Important (dent triangulaire) \\
\hline \multicolumn{3}{|l|}{ Examen du parodonte } \\
\hline Mobilité & Physiologique ou réduite & Importante \\
\hline Récession & Absente & Importante \\
\hline \multicolumn{3}{|l|}{ Examen de l'occlusion } \\
\hline - Statique & $\begin{array}{l}\text { Classe I, classe II div.1, } \\
\text { Classe III, Béance ou bout à bout }\end{array}$ & Supraclusion importante \\
\hline Dynamique & Fonction Canine & \\
\hline Facettes d'usure & Absente & Présente \\
\hline - Plan d'occlusion & & Perturbée \\
\hline
\end{tabular}


faveur de la prothèse collée au dépens de l'implant peut être emporté par :

- des conditions anatomiques défavorables (faiblesse du volume osseux) ;

- une impossibilité médicale de la pose implantaire ;

\section{Cas clinique}

\section{Examen clinique}

Il s'agit d'un patient âgé de 24 ans qui consulte dans le but de remplacer la 24 perdue pour raison carieuse. Il est en bon état de santé générale. Le patient nous fait part de son refus d'une solution amovible tout en insistant sur le désir d'une restauration esthétique durable.

L'examen clinique objective un bon niveau d'hygiène orale. L'examen des dents bordant
- un souhait du patient cantonné sur des techniques moins invasives ;

- un choix économique.

I'édentement révèle que la 23 est saine, cependant la 25 présente une petite carie mésiale. Ces deux dents sont en normoposition. Leurs indices mésio-distaux (Le Huche) sont peu élevés et leurs hauteurs coronaires satisfaisantes (fig. 4 a et b).

La zone édentée est recouverte d'une fibromuqueuse ferme et adhérente, et présente une petite perte de substance ostéo-muqueuse de classe I (classification de Siebert, 1983). La
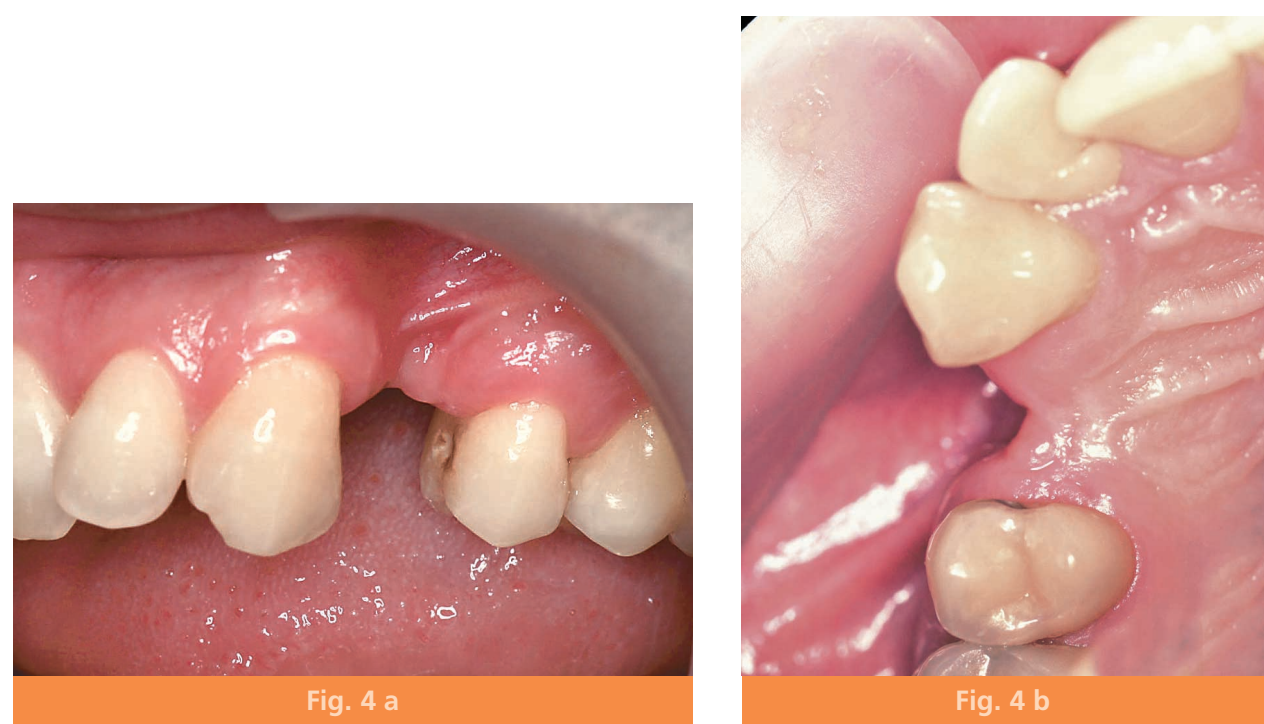

Fig. 4 a et b État initial d'édentement unitaire ;

a : vue vestibulaire

b : vue occlusale. La 23 est saine, la 25 présente une petite carie mésiale. Ces dents sont en normopostion. La zone édentée est recouverte d'une fibromuqueuse saine et présente une petite perte de substance ostéo-muqueuse de classe I (classification de Siebert 1983). 


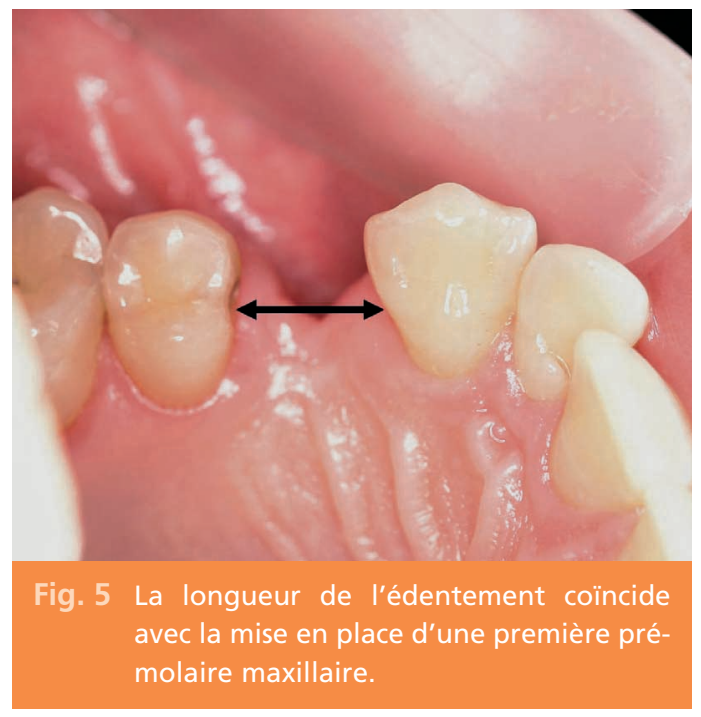

longueur de l'édentement coïncide avec la mise en place d'une première prémolaire maxillaire (fig. 4 a, 5).

L'occlusion d'intercuspidation maximale est stable. En propulsion, on observe un guide incisif efficient. En diduction droite et gauche, on constate une fonction canine efficace (fig. 6).

\section{Décision thérapeutique}

Quatre possibilités thérapeutiques s'offrent à nous pour compenser cet édentement unitaire : prothèse amovible partielle, prothèse implanto-portée, prothèse partielle collée et un bridge conventionnel.

La solution amovible est d'emblée rejetée compte tenu des doléances du patient. La solution implantaire est écartée pour des raisons financières. Le délabrement excessif des dents piliers lors de la réalisation d'un bridge conventionnel nous incite à privilégier une solution plus conservatrice, en l'occurrence un bridge collé.

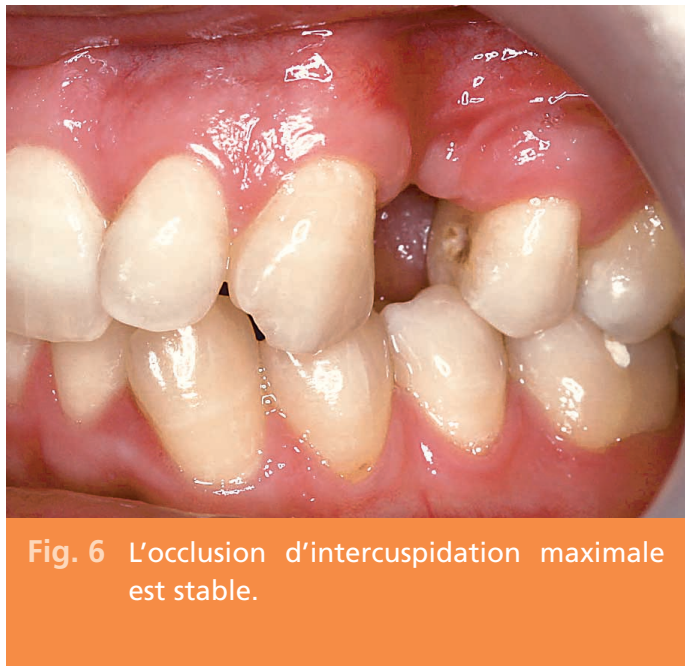

\section{Séquences prothétiques}

Comme en prothèse conventionnelle, la préparation doit répondre à des impératifs mécanique, esthétique et biologique.

Bien que le mode de liaison par collage assure une rétention importante, grâce à l'adhésion chimique sur l'émail, et autorise des préparations moins mutilantes, ces préparations doivent assurer rétention, sustentation et stabilisation $[9,10]$ (fig. 7 a et b).

La rétention sera assurée par la mise de dépouille des faces proximales des dents en fonction de l'axe d'insertion, par les moyens secondaires de rétention : ce sont principalement les rainures proximales, elles doivent être parallèles à l'axe d'insertion et ont une forme cylindro-conique avec un méplat cervical. Pour la 25, la carie proximale a été englobée dans la préparation sous forme d'une boîte proximale ce qui dispense de toute éventuelle rainure.

La sustentation est assurée par une limite cervicale en forme de congé, par le fond plat des rainures ou des boîtes proximales. La stabilisation nécessite un ceinturage de plus de $180^{\circ}$, 

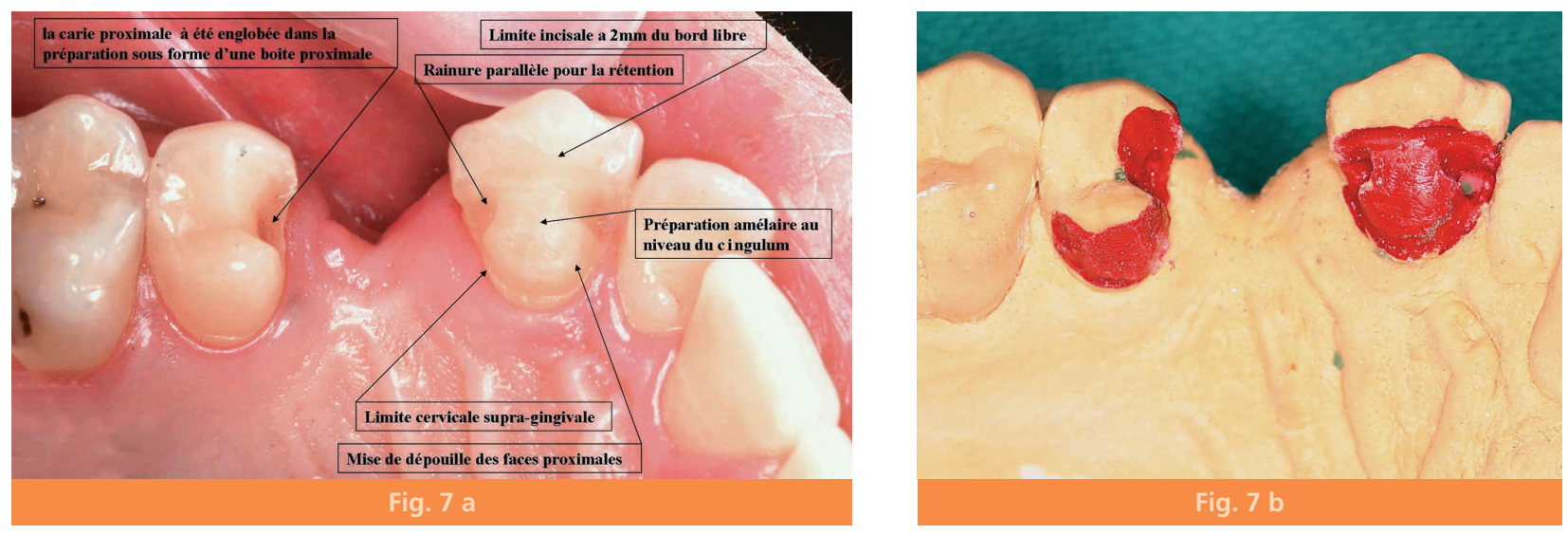

Fig. 7 a et b Principes de préparations pour bridge collé.

cependant pour des raisons esthétiques la préparation se situe en retrait des jonctions proximo-vestibulaires. Ceci diminue le ceinturage et accroît la nécessité des rainures proximales.

En outre, pour des impératifs biologiques liés au collage, la préparation doit être supra-gingivale. De même, elle doit être suffisante pour permettre la mise en place d'une armature métallique de 0,3 à 0,4 mm d'épaisseur qui assurera une résistance mécanique à la prothèse sans engendrer de sur-contour.

Au niveau occlusal, le joint dento-prothétique ne doit pas être situé au niveau d'un point de contact d'occlusion dynamique ou statique, sous peine de détériorer le joint de colle et de risquer une infiltration bactérienne.

Enfin, la préparation doit permettre de préserver I'aspect esthétique [11]. Les limites de la préparation doivent être déterminées de façon à ce que le métal ne soit pas visible : les rainures proximales doivent être judicieusement situées en retrait des jonctions proximovestibulaires et les limites incisales à $2 \mathrm{~mm}$ du bord libre.

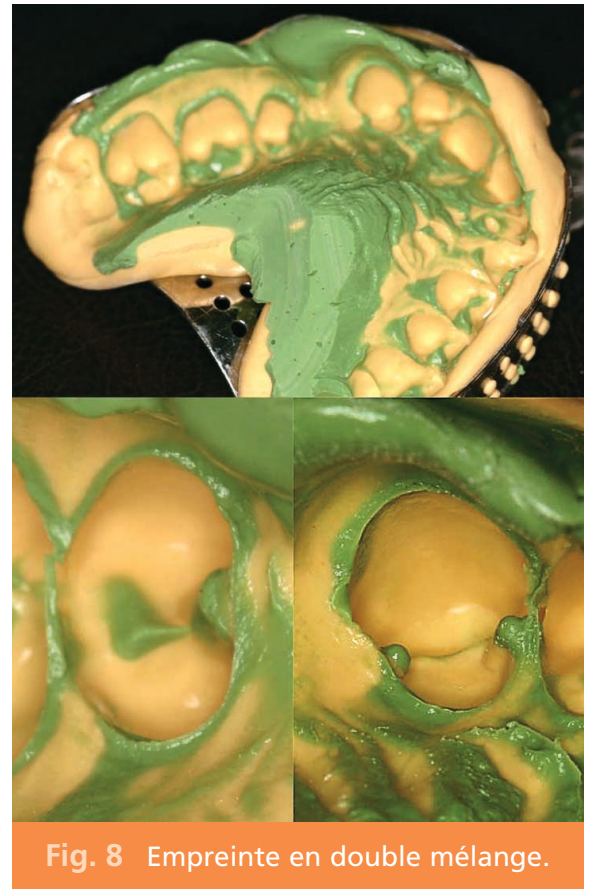

La prise d'empreinte ne présente pas de difficulté, les limites étant toutes supragingivales. Une technique en un temps sera préconisée (fig. 8).

L'essayage permet de vérifier l'ajustage dento-prothétique, l'absence de bascule et l'intégration esthétique de l'intermédiaire de la prothèse (fig. 9 a à $\mathbf{c}$, fig. 10 a et b). 
La friction de la pièce prothétique doit être légère car la prothésiste doit avoir prévu une épaisseur suffisante pour le joint de colle.
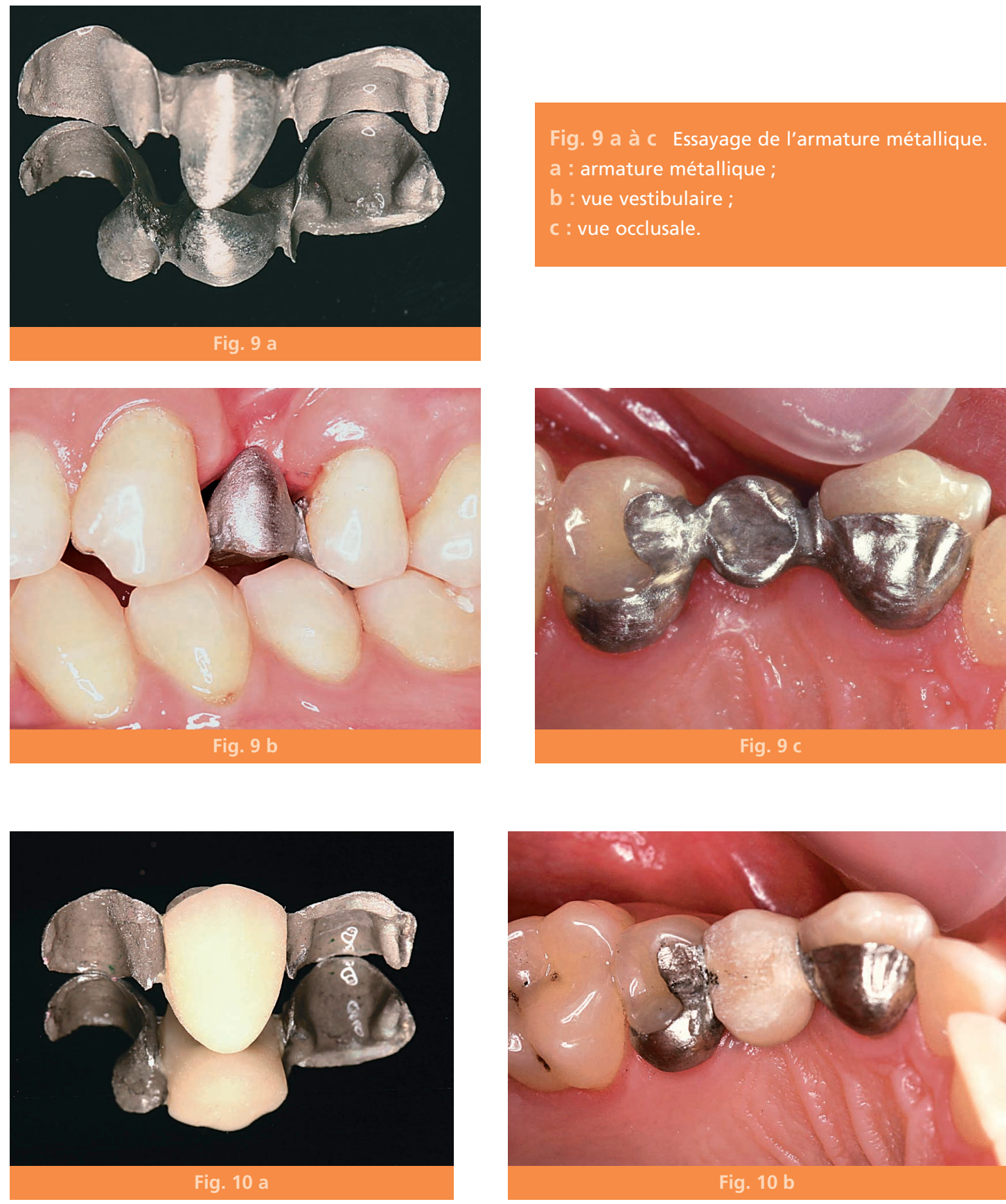

Fig. 10 a et b Essayage a l'état du biscuit.

a : bridge à l'état de biscuit ; b : essayage et réglage de l'occlusion.
Le collage est réalisé sous champ opératoire selon les consignes du fabricant (une résine adhésive Superbond est préconisée).

Fig. 9 a à c Essayage de l'armature métallique.

a : armature métallique ;

b : vue vestibulaire ;

c : vue occlusale. 

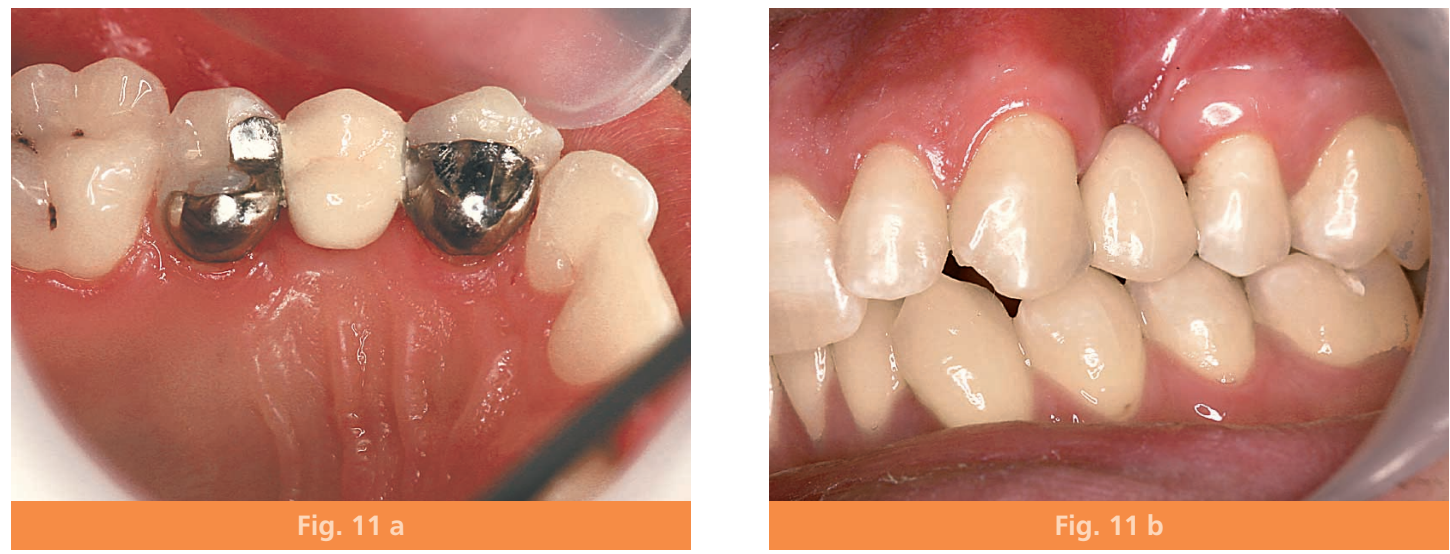

Fig. 11 a et $b$ Résultat final.

a : vue occlusale ; $b$ : vue vestibulaire.

Après dépose du champ opératoire, les ailettes métalliques ne doivent pas être visibles lors du sourire. Les dents piliers ne présentent aucun assombrissement grâce à l'opacité de la colle. Les embrasures dégagées permettent l'entretien avec les brossettes interdentaires (fig. 11 a et b).

\section{Conclusion}

La compensation d'un édentement unitaire par bridge collé permet d'apporter une solution répondant aux impératifs fonctionnels, biologiques et esthétiques. De plus, ce type de restauration semble être garant d'une bonne longévité en regard des données de la littérature.
Seule une analyse précise du contexte clinique permet d'aboutir au choix final raisonné de la meilleure option thérapeutique. Actuellement, dans les limites de leurs champs d'application, leur intérêt dans notre arsenal thérapeutique apparaît maintenant incontournable.

\section{Bibliographie}

1. Folch $\mathrm{H}$, Abgral S, Armand S, Verge J.

Bridges collés. Encycl Méd Chir Odontologie. 23280-A-10.

2. Dunne SM, Millar BJ. A longitudinal study of the clinical performances of resin-bonded bridges and splints.

Brit Dent J 1993;174: 405-411.

3. Priest G.F. Esthetic comparison of alternatives for remplacement of single missing tooth. J Esthet Dent 1996;8:58-65.

4. Lehman N, Simon A-L, Tirlet G. Édentement unitaire : 
de l'observation clinique

à la prise de décision

thérapeutique.

Rev Odonto Stomat

2006;35:33-61.

5. Shillingburg HT.

Bases fondamentales

en prothèse fixée.

$3^{e}$ ed.,

Vélizy-Vallacoublay,

CdP Initiatives Santé 1998:512.

6. Assémat-Tessandier $X$.

\section{Modifications}

des dents

supports d'ancrage

des bridges collés.

Cah Prothèse 1986;54:47-60.
7. Brodeur $C$, Prevost AP, Boudrias $P$, Helie $P$, de Grandmont $P$,

Allaire J.

Le pont papillon : causes de descellement. J Dent Québec 1990;27:217-224.

8. Creugers NHJ, Snoek PA, Van'hot MA, Kayser AF. Clinical performance of resin bonded: 5 years prospective study. Part III.

Failure characteristics and survival after rebondede. J Oral Rehab 1990;17:179-186.
9. Brabant A.

Méthodologie

clinique

de préparations

pour bridges collés.

Réalités cliniques

1996; vol 7(4):513-521.

10. Brabant A.

La rétention

des bridges collés.

Cahiers de Prothèse

1995;92:2-11.

11. Brabant A.

Esthétique et fiabilité

des bridges collés.

Rev Odont Stomat

1993;22:387-395.

\section{SUMMARY}

\section{The unitary toothless: \\ rehabilitation with a bonded bridge}

Hicham SOUALHI

Amal EL YAMANI

Jamal EL BERNOUSSI

\section{Keywords}

- unitary toothless

- bonded bridge

- therapeutical decision
The unitary toothless situations represent one of the clinical situations witch offer the most large therapeutical eventails.in effect, however the concerned sector, the treatment hypotheses are Nemours :implanto-hold prosthesis, celled bridge, conventional bridge, movable prosthesis, and also orthodontical treatment.

In the limits of their indication's field, the celled bridge constitutes a choice alternative answering to functional, esthetical and biological imperatives.

Essentially, they address for cases of unitary toothless; borded by the normoposition's teeth, nearly healthy and reduced mobility. Their indication's rigor constitutes an important element in their successfulness. 